\title{
Predicting Foot Positions for Manual Materials Handling Tasks
}

\author{
David W. Wagner, Matthew P. Reed, and Don B. Chaffin \\ University of Michigan
}

Copyright @ 2005 SAE International

\begin{abstract}
For many industrial tasks (push, pull, lift, carry, etc.), restrictions on grip locations and visibility constrain the hand and head positions and help to define feasible postures. In contrast, often the foot locations are minimally constrained and an ergonomics analyst can choose several different stances in selecting a posture to analyze. Also, because stance can be a critical determinant of a biomechanical assessment of the work posture, the lack of a valid method for placing the feet of a manikin with respect to the task compromises the accuracy of the analysis. To address this issue, foot locations and orientations were captured in a laboratory study of sagittal plane and asymmetric manual load transfers. A pilot study with four volunteers of varying anthropometry approached a load located on one of three shelves and transferred the load to one of six shelves. The data illustrate foot placements and behaviors that depend on pickup heights, the use of one or two hands to grasp the object, and the participants' body dimensions. Two distinct pickup and delivery strategies were observed. Split stance, with one foot in front of the other, was markedly more frequent than parallel stance with the feet side by side. A statistical model was developed to predict foot placements at load pickup. This study confirms the importance of this topic and provides the basis for the much more comprehensive study that is now underway.
\end{abstract}

\section{INTRODUCTION}

Ergonomic analysis is a common application of digital human models (DHM). Analysis tools readily available in commercial DHM software include the NIOSH lifting equation, the University of Michigan 3D Static Strength Prediction Program, low-back analyses, and joint torque analyses. All of these ergonomic tools require as input a posture (usually static) and a set of subject and task parameters such as body dimensions and hand forces. Input postures for these programs are typically created using motion capture, manual manikin manipulation, or using a posture prediction algorithm. Motion capture provides the most accurate postures, but only for the individual for whom data are available. Manual manikin manipulation can produce an arbitrary posture with unknown accuracy. Statistical posture prediction models produce postures that have quantifiable accuracy on particular aspects of the posture, but are limited by the range of data on which the models were developed.

One of the most important considerations in the prediction of posture in standing tasks is the placement of the feet. Currently, no general models for prediction of foot positions for manual materials handling tasks are available in the open literature.

Many experimental investigations into lifting limits and behaviors have focused on sagittal plane lifting and to a lesser extent asymmetric lifts that require no foot movements. A study by Baril-Gingras et al. (1995) focused on the handling strategies of objects other than boxes in a distribution center for a large transport company. Of the 944 handlings that were catalogued, workers took two or more steps in over half $(57 \%)$. Furthermore, approximately $77 \%$ of the movements documented by Baril-Gingras et al. included some type of horizontal component, as opposed to a strictly vertical exertion or sagittal plane lift. The study confirms the common observation that most materials handling tasks in industry require workers to take one or more steps, and that few tasks are performed solely in the sagittal plane. Yet, these more complex stepping tasks are not well represented in the biomechanical literature, and hence the ergonomist has little guidance for predicting appropriate postures in DHM software.

Several predictive models have been developed to generate general postures for manual material handling tasks (Andres, 1991; Ayoub and Lin, 1995; Ayoub 1998; Dysart and Woldstad, 1996). Depending on the hand load involved, these models have shown a great sensitivity to the load-feet distances. However, foot placements are usually a required input to the models (3D Static Strength Prediction Program, University of Michigan, 2002; Ayoub and Lin, 1995; Ayoub, 1998; Dysart and Woldstad, 1996; Chaffin and Baker, 1970). For many ergonomic analyses of materials handling 
tasks, the location of the object to be moved is known in relation to the floor along with other task and environmental constraints, but the foot placements a worker would choose to lift the object are not known.

Throughout this paper, the terms "parallel" and "split" will be used to describe stance (foot placement). In parallel stance, the feet are not separated in the anterior or posterior direction but are approximately side-by-side. Parallel stance has been described by others as the posture taken when the instruction is given for the feet to be "shoulder width apart." Split stance is defined as anything that is not parallel stance. Split stance is most widely seen as the foot placement during the double support phase of walking.

Kollmitzer et al. (2002) compared the advantages of parallel and split stance for selected lifting tasks. The results were inconclusive as both stances exhibited positive and negative aspects. Split stance was observed to reduce the complexity of balance control in the anterior/posterior direction but increased the susceptibility to lateral perturbations. During lifting tasks, the available postural control tactics are dependent on foot placement. This paper presents an initial investigation of foot placements in standing load transfer tasks. A statistical analysis of the foot positions of four participants in a laboratory study demonstrates the feasibility of predicting foot positions from load and subject variables.

\section{METHODS}

\section{Facility and Test Conditions}

Data for the current analysis were obtained as part of a larger study of load transfer motions conducted in the Human Motion Simulation Laboratory (HUMOSIM) at the University of Michigan. Figure 1 shows a participant in the test facility performing a transfer trial. Testing was conducted with two three-shelf towers with low, medium, and high shelves. The two towers were oriented at right angles and placed $1.06 \mathrm{~m}$ apart.

Data were obtained from two men with statures of 183 $\mathrm{cm}$ and $174 \mathrm{~cm}$ and two women with statures of $164 \mathrm{~cm}$ and $151 \mathrm{~cm}$. All participants were right-hand dominant young adults ranging in age from 19 to 28 years. Participants with low body mass index (median 22.3 $\mathrm{kg} / \mathrm{m}^{2}$, maximum $27.2 \mathrm{~kg} / \mathrm{m}^{2}$ ) were selected to facilitate placement of the sensors and tracking of the underlying skeletal structures. Because of the small number of participants, the sample cannot be used to create a general model for industrial workers. However, the sample may be adequate to quantify typical foot behaviors, thus justifying a much larger study, and assess the usefulness of using regression equations to predict foot positions during common load transfer tasks.
A balanced presentation of the full factorial test conditions was constructed for each subject. Test variable levels were three pickup shelves (tower 1 ), six delivery shelves (tower 1 and tower 2), three carry hand conditions, and two load weights. The two towers had equivalent shelf heights. Low, middle, and high shelves measured from the floor were set at $18 \mathrm{~cm}, 89 \mathrm{~cm}$, and $166 \mathrm{~cm}$ respectively. The low, middle, and high shelves were the same for all participants except for the participant with the shortest stature, for whom the high shelf on each tower was lowered to $161 \mathrm{~cm}$ to accommodate her range of motion. The single-handed light and heavy loads used were $2.27 \mathrm{~kg}$ (5 lbs) and 4.54 $\mathrm{kg}(10 \mathrm{lbs})$ and the two-handed light and heavy loads used were $4.54 \mathrm{~kg}$ (10 lbs) and $9.07 \mathrm{~kg}$ (20 lbs).

Each participant was tested with each load weight, carry hand(s), and shelf combination for a total of 90 transfer trials. After receiving an auditory signal, the participant approached, picked up, and delivered a load to a different shelf. Prior to each trial, the participant was instructed with which hand(s) (left, right, both) to pick up the load, which shelf to deliver the load, and reminded of the load weight (light or heavy). Each participant was instructed to begin the approach toward the load with the right foot. This instruction was used to limit the observed stances to either split stance with the contra-lateral leg to the delivery tower as the trailing limb or parallel stance. Trials in which the participant started with the left foot were omitted from the analysis.

Prior to data collection, each participant practiced performing similar load transfer trials for 20 minutes to become familiar with protocol and to learn the task. Participants were instructed to self-select their start position at the beginning of the experiment to be approximately three steps from tower 1 . The starting foot locations were outlined on the floor and used for all trials. Figure 2 shows the test facility dimensions.

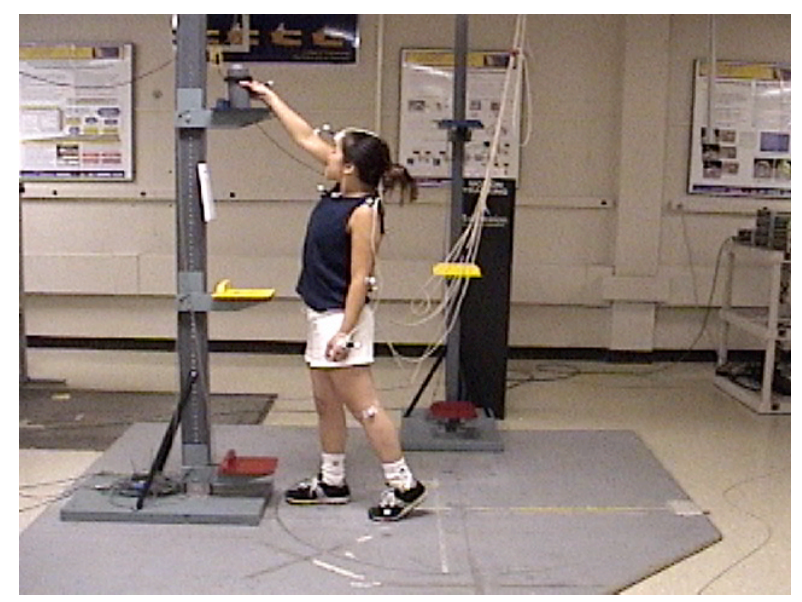

Figure 1. Participant in test facility layout, shelving tower 1 and 2 , and motion capture hardware. Participant is shown at tower 1. 


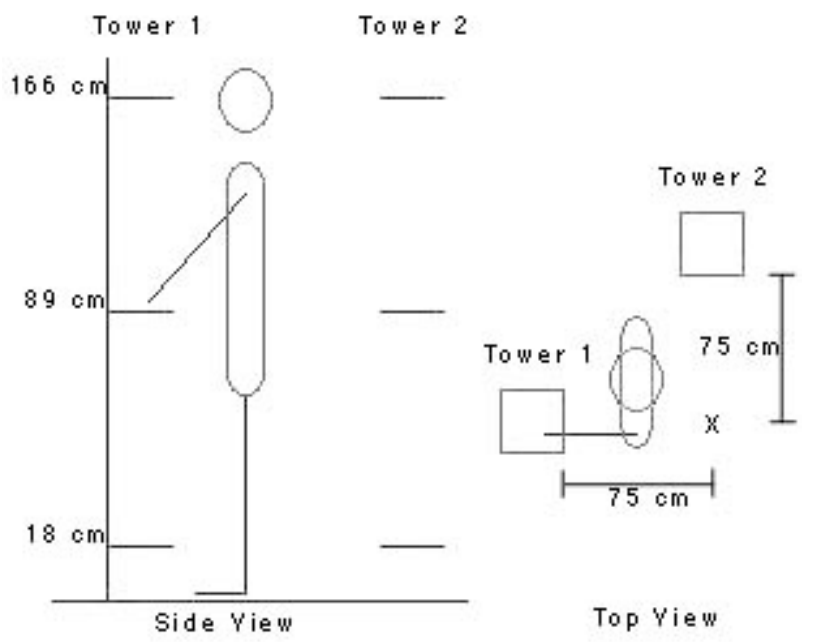

Figure 2. Test facility shelf layout showing shelving tower 1 (pickup tower) and tower 2 dimensions.

\section{Motion Capture}

Whole-body motions were recorded using a combined electromagnetic Flock of Birds (Ascension Technologies) and optical PCReflex (Qualisys) system. Each Flock of Birds (FOB) sensor reports six degrees of freedom (position and orientation) while only position is obtained for each retro-reflective marker used by the optical system. FOB sensors were placed on the forehead, over the T8, sacrum, and sternum body landmarks, and on the back of the right and left hands. Optical markers were affixed to the FOB sensors for position measurement redundancy and to take advantage of the increased resolution provided by the optical system. Additional optical markers were placed on the left and right acromion process, lateral epicondyle of the humerus, lateral epicondyle of the femur, lateral malleolus, and the fifth-metatarsal phalangeal body landmarks.

Prior to testing, a probe FOB sensor was used to digitize body landmarks on the forehead, torso, pelvis, and arms. Optical markers and FOB sensors were recorded simultaneously with the probe location to define each body landmark location in a relevant body fixed frame of reference defined by marker and sensor locations. Transformation matrices were constructed from the digitization data to reference the optical markers and FOB sensors to anatomically based coordinate systems for each body segment using relationships described in Reed et al (1999).

Data were sampled from each system at $25 \mathrm{~Hz}$ during the trial. Switch sensors on each tower shelf activated by a change in weight on the shelf were used to obtain the time of load pickup and delivery during each trial. Joint centers and body landmarks were calculated from the data and used in a 14-link biomechanical model to determine the projected Center of Mass (COM) position. The biomechanical linkage uses the deLeva representation of the Zatsiorsky segmental inertial parameters (deLeva 1996).

\section{RESULTS}

\section{Foot placements and behaviors}

Foot positions and orientations at the time of load pickup were analyzed. Linear regression models were constructed to predict the foot placements. Figure 3 shows a depiction of the dependent variables predicted by the regression models. The dependent measures were chosen as a representation of the three degrees of freedom $(x, y, \Theta)$ for each foot. The position of the projection of the Center of Mass onto the floor was chosen as a representation of the whole body position. Heel and toe raises can be considered fourth and fifth degrees of freedom for the foot but were not addressed here.

Table 1 lists the subject and task parameters used as potential predictors in the regression analyses. The independent measures are selected to be among those readily available in digital human modeling environments.

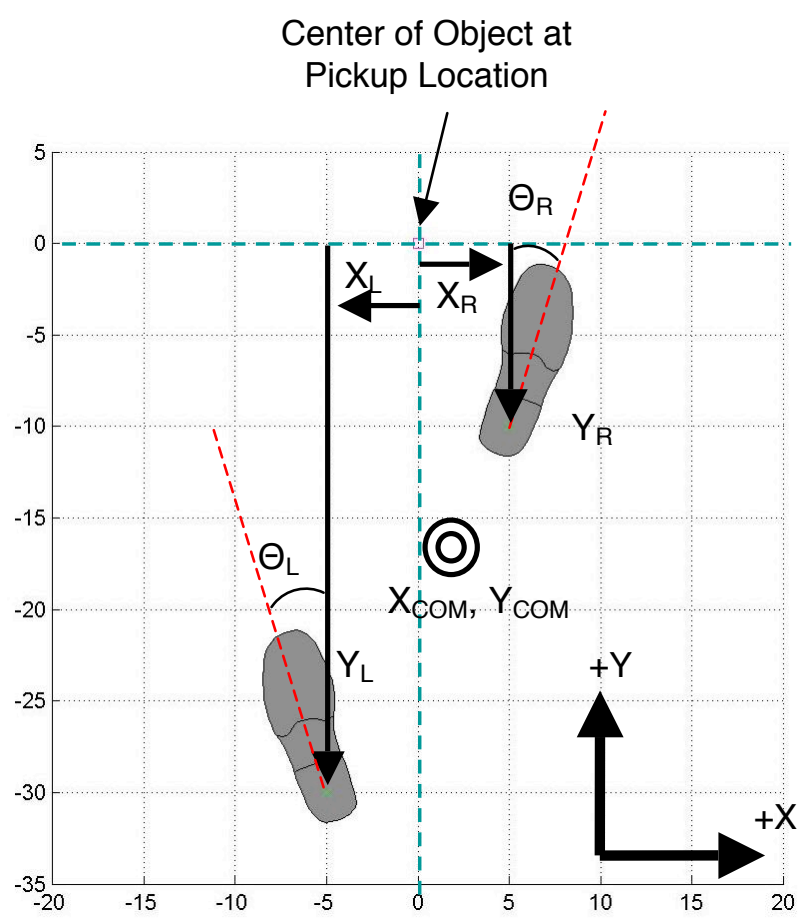

Figure 3. Parameterization of dependent measures with respect to load position. $X_{R}, Y_{R}, \Theta_{R}, X_{L}, Y_{L}, \Theta_{L}, X_{C O M}$, $Y_{\text {COM }}$, correspond to the horizontal right heel position, vertical right heel position, right foot orientation, horizontal left heel position, vertical left heel position, left foot orientation, horizontal center of mass position, and vertical center of mass position respectively.

The predicted fore-aft distance of the right heel from the load, defined here as $Y_{R}$, has an adjusted $R^{2}$ value of 0.52 based on subject stature alone. Additional 
predictors did not have a significant effect on increasing the predictive capability of the right foot vertical distance.

The hand(s) (left, right, both) used to transfer the load significantly affected the horizontal distance of the right heel, defined here as $X_{R}$, with respect to the load. The adjusted $R^{2}$ value for predicting the right heel medial/lateral position is 0.58 . Figure 4 shows the $90 \%$ confidence interval of medial/lateral heel placements used for the different carry hand(s). The right heel was significantly farther to the right of the load when the left hand was used to pick up the load than when the load was lifted with the right hand or both hands.

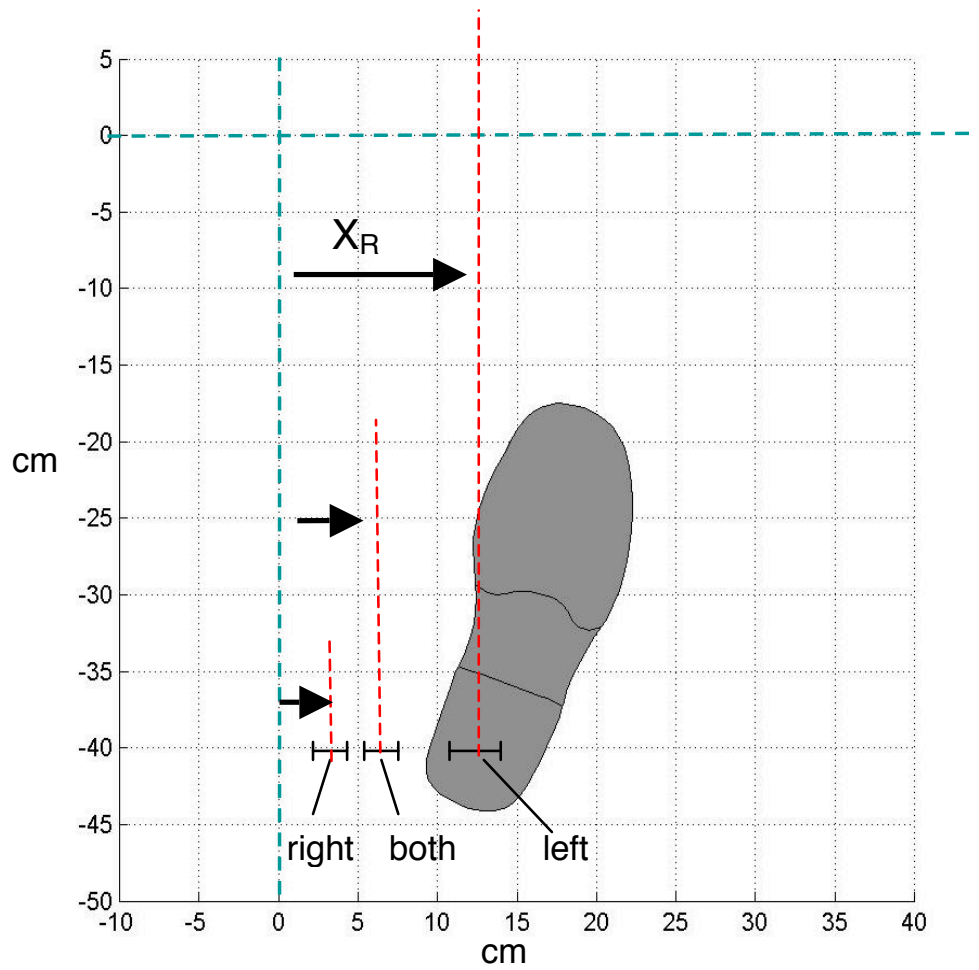

Figure 4. Leading leg horizontal position partitioned by load pick up hand(s). Ninety percent confidence intervals (+/- 1.64 standard deviations) are centered on the mean lateral heel position for the respective pick up hand(s).

Table 1 summarizes the task and subject variables that were significant $(p<0.05)$ predictors of foot position and orientation. The table also includes the fore-aft and lateral position of the projected center of mass of the body, labeled as $Y_{\text {сOM }}$ and $X_{\text {Com }}$ respectively. The dependent variables are ordered by adjusted $R^{2}$ value. Interestingly, load weight did not significantly affect any of the dependent measures. However, the predicted variables account for more than half of the variance in lateral COM position and lead foot position, lending support for the use of simple regression equations to predict foot and stance behavior for terminal postures during load transfer tasks.
Table 1

Results of Linear Regression Analysis

\begin{tabular}{|c|c|c|c|c|c|c|}
\hline Dependen & Root & & Indepen & dent Vari & ables & \\
\hline |variabie & $\begin{array}{l}\text { Squean } \\
\text { Square } \\
\text { Error }\end{array}$ & $\#$ & $\begin{array}{l}\text { Load } \\
\text { Carry } \\
\text { Hand(s) }\end{array}$ & Stature & $\begin{array}{l}\text { Pickup } \\
\text { Height }\end{array}$ & $\begin{array}{l}\text { Load } \\
\text { Weight }\end{array}$ \\
\hline$x_{\text {сом }}$ & 3.61 & 0.67 & $x^{*}$ & $x$ & $x$ & \\
\hline$\Theta_{\mathrm{R}}$ & 7.60 & 0.65 & $x$ & $x$ & $x$ & \\
\hline$X_{R}$ & 3.66 & 0.53 & $x$ & & & \\
\hline$Y_{R}$ & 4.83 & 0.52 & & $x$ & & \\
\hline$X_{L}$ & 4.46 & 0.38 & $x$ & $x$ & & \\
\hline$Y_{\mathrm{COM}}$ & 5.37 & 0.37 & $x$ & $x$ & $x$ & \\
\hline$Y_{L}$ & 10.18 & 0.19 & & $x$ & & \\
\hline$\Theta_{L}$ & 8.73 & 0.16 & $x$ & $x$ & $x$ & \\
\hline
\end{tabular}

${ }^{*} x$ indicates that the factor was significant with $p<0.05$.

\# The dependent variables are ordered by adjusted $R^{2}$ value

\section{DISCUSSION}

This pilot study demonstrated that foot placements in load transfer tasks can be predicted to a useful level of accuracy with fairly simple statistical models. Lateral $\mathrm{COM}$ and lead foot position and orientation are the bestpredicted variables, while fore/aft COM and trailing leg position and orientation are not as well explained. Lateral lead foot placement was well predicted using only the carry hand(s). Trailing leg placement shows a higher residual variability than other dependent variables, suggesting differing stance behaviors between subjects. Similar reasoning also explains the variability of the fore/aft COM position as the fore/aft trailing leg and COM positions are related. The study provides insights for realistic foot placements in simulating noncyclical stepping behavior in the work cell environment.

The generality of this study is limited in several important ways. The small sample size of young, fit participants limits the applicability of the results. The limited range of task conditions also limits the predictive capacity to similar direct approach pickup and 90-degree right turn delivery tasks. The participants were also required to maintain ground contact with the left foot after the load was first lifted, which may have limited the range of foot placement behaviors. However, the results suggest that a more comprehensive investigation could produce a statistical model that would be useful for posturing figure models used to analyze standing tasks.

A new study now underway will provide a more generalizable and robust model. Additional approach and delivery vectors spanning 90 and 200 degrees respectively are being used. A larger range of weights is also being used to address the interesting absence of a significant effect of load weight. Force plate data collected at pickup and delivery will support biomechanical analysis of stepping behaviors. The 
ultimate goal is to develop a model that accurately simulates non-cyclical work cell stepping behaviors for not only load transfers but for other material handling tasks as well. More accurate stepping predictions will improve the validity of posture prediction in human figure model software, thereby improving the accuracy of ergonomic analyses.

\section{ACKNOWLEDGMENTS}

This research was sponsored by the partners of the Human Motion Simulation (HUMOSIM) program at the University of Michigan. HUMOSIM partners include DaimlerChrysler, Ford, General Motors, International Truck and Engine, United States Postal Service, U.S. Army Research and Development Engineering Command (RDECOM), and Lockheed Martin.

\section{REFERENCES}

Andres, R. O. and D. B. Chaffin (1991). Validation of a biodynamic model of pushing and pulling. Journal of Biomechanics 24(11): 1033-45.

Authier, M., M. Gagnon, et al. (1995). Handling Techniques: The Influence of Weight and Height for Experts and Novices. International Journal of Occupational Safety and Ergonomics 1(3): 262-275.

Authier, M., M. Lortie, et al. (1996). Manual handling techniques: Comparing novices and experts. International Journal of Industrial Ergonomics 17(5): 419-429.

Ayoub, M. M. (1998). A 2-D simulation model for lifting activities. Computers \& Industrial Engineering 35(3-4): 619-622.

Ayoub, M. M. and C. J. Lin (1995). Biomechanics of manual material handling through simulation: Computational aspects. Computers \& Industrial Engineering 29(1-4): 427-431.

Baril-Gingras, G. and M. Lortie (1995). The Handling of Objects Other Than Boxes: Univariate Analysis of Handling Techniques in a Large Transport Company. Ergonomics 38(5): 905-925.

Center for Ergonomics, Three Dimensional Static Strength Prediction Program (3DSSPP), The University of Michigan, 2002.

Chaffin, D. B. and W. H. Baker (1970). A biomechanical model for analysis of symmetric sagittal plane lifting. American Institute of Industrial Engineers Transactions 2(1): 16-27.
deLeva, P. (1996). Adjustments to ZatsiorskySeluyanov's segment intertia parameters. Journal of Biomechanics 29(9): 1223-1230.

Delisle, A., M. Gagnon, et al. (1996). Load Acceleration and Footstep Strategies in Asymmetrical Lifting and Lowering. International Journal of Occupational Safety and Ergonomics 2(3): 185-195.

Delisle, A., M. Gagnon, et al. (1998). Knee flexion and base of support in asymmetrical handling: effects on the worker's dynamic stability and the moments of the L(5)/S(1) and knee joints. Clinical Biomechanics (Bristol, Avon) 13(7): 506-514.

Delisle, A., M. Gagnon, et al. (1999). Kinematic analysis of footstep strategies in asymmetrical lifting and lowering tasks. International Journal of Industrial_Ergonomics 23(5-6): 451-460.

Drury, C. G., C.-H. Law, et al. (1982). A survey of industrial box handling. Human Factors 24(5): 553-565.

Dysart, M. J. and J. C. Woldstad (1996). Posture prediction for static sagittal-plane lifting. Journal of Biomechanics 29(10): 1393-7.

Gagnon, M., A. Plamondon, et al. (1993). Pivoting with the load. An alternative for protecting the back in asymmetrical lifting. Spine 18(11): 1515-24.

Holbein, M. A. and D. B. Chaffin (1997). Stability limits in extreme postures: effects of load positioning, foot placement, and strength. Human Factors 39(3): 456-68.

Holbein, M. A. and M. S. Redfern (1997). Functional stability limits while holding loads in various positions. International Journal of Industrial Ergonomics 19(5): 387-395.

Kollmitzer, J., L. Oddsson, et al. (2002). Postural control during lifting. Journal of Biomechanics 35(5): 585-94.

Reed, M.P., Manary, M.A., and Schneider, L.W. (1999). Methods for measuring and representing automobile occupant posture. Technical Paper 990959. SAE Transactions: Journal of Passenger Cars, Vol. 108.

Waters, T.R., Putz-Anderson, V., Garg, A., Fine, L.J. (1993). Revised NIOSH equations for the design and evaluation of manual lifting tasks. Ergonomics, 36, 749776.

Zatsiorsky, V. and Seluyanov, V. (1983). The mass and intertia characteristics of the main segments of the human body. In: Matsui, H. and Kobayashi, K., Editors, 1983. Biomechanics VIII-B, Human Kinetic, Illinois, 1152-1159. 